\title{
Official American Thoracic Society/American College of Chest Physicians clinical practice guideline: Liberation from mechanical ventilation in critically ill adults
}

\author{
Kenneth Nugent MD, Hawa Edriss MD
}

The American College of Chest Physicians and the American Thoracic Society have recently published clinical guidelines on the management of mechanical ventilation in critically ill adult patients who are near extubation. These guidelines considered six important questions in the management of these patients. ${ }^{1-3}$

1. Should spontaneous breathing trials be conducted with or without inspiratory pressure support?

This panel suggested that spontaneous breathing trials should be conducted with inspiratory pressure support in the range of $5-8 \mathrm{~cm}$ of water. This approach can increase extubation success from approximately $67 \%$ to $74 \%$. This information is based on four randomized controlled trials which included 875 patients.

The exact reason for this improvement in outcomes is unclear. It may be that clinicians underestimate the work of breathing associated with endotracheal tubes and that even short periods of spontaneous breathing through endotracheal tubes can cause critical respiratory muscle fatigue in some patients. Spontaneous breathing trials may also trigger dyspnea and then anxiety which reduces the ability of the patient to cooperate with the trial and proceed to successful extubation.

We recommend pressure support (PS) at $5 \mathrm{~cm}$ of water and PEEP at $5 \mathrm{~cm}$ of water prior to extubation. This pressure supports the respiratory muscles, overcomes the resistance generated by the endotracheal tube, and reduces anxiety. This strategy allows clinicians a longer time to assess patients and make decisions

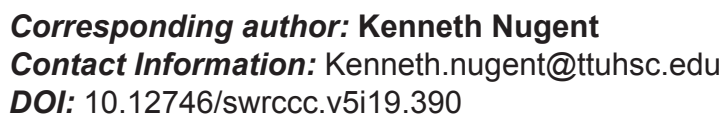

about extubation. We have worked with intensivists who start with a PS of 5-10 $\mathrm{cm}$ of water and decrease it to zero as the patients adapt to the new settings. Careful institution of a T-piece or the application of no PS can give clinicians more confidence about the decision to extubate if this approach tolerated. However, a T-piece should not be routinely used, and if it is, it should be for a limited time, say 2-3 minutes.

2. Do protocols to minimize sedation in mechanically ventilated patients affect the duration of ventilation, the duration of ICU stay, and the short-term mortality?

This panel concluded that sedation protocols do not affect the duration of mechanical ventilation or short term mortality but do reduce ICU length of stay. This conclusion was based on six randomized controlled trials with 1059 patients. The absolute mean difference was one day (95\% confidence interval [Cl]: 2.14 fewer days to 0.14 more days). They noted that the evidence supporting the conclusions about the duration of mechanical ventilation and length of stay was very low quality.

We recommend using a systematic approach and outlining the general principles of analgesia and sedation to ICU nurses, training physicians, and other clinicians to provide a balanced approach to optimal patient comfort and care while limiting the risk of complications and cost.

In our opinion, protocols do help to achieve an organized and efficient work environment, especially in situations in which clinicians get frequently overworked, such as in critical care units. However, flexibility with protocol use should be allowed and changes should be based on assessment by the responsible clinician to account for the high variability in ICU patients' characteristics and underlying comorbidity. 
3. Does extubation to noninvasive ventilation in high risk patients have a favorable effect on extubation success?

This panel concluded that extubation to noninvasive ventilation increased the extubation success from $77 \%$ to $88 \%$. This information was based on randomized control trials which included 525 patients. This strategy also reduces the ICU length of stay and short-term mortality. Risk factors for extubation failure included COPD, congestive heart failure, hypercapnia, older age, and a higher severity of illness score. Previous studies concluded that reintubation causes increased mortality and morbidity, including deconditioning, critical care polyneuropathy, nosocomial pneumonia, less successful next extubation attempts, higher costs, and longer ICU and hospital lengths of stay.

We recommend the immediate institution of non-invasive ventilation in high risk patients who tolerated spontaneous breathing trials as suggested by the evidence mentioned above. It is the intensivist's duty to predict and identify patients at high risk for extubation failure and to start non-invasive mechanical ventilation early after extubation.

4. Should patients requiring mechanical ventilation for more than 24 hours participate in a protocol for rehabilitation directed at early mobilization?

The panel concluded that these patients should participate in the protocol for rehabilitation. Benefits included a shorter duration of mechanical ventilation (2.7 fewer days; $95 \% \mathrm{Cl}: 4.2$ to 1.2 fewer days). This result was based on one randomized control trial with 104 patients. Adverse events associated with these protocols seem to be minimal. However, there is no definite conclusion as to who should participate, when the participation should start, and what the program should be.

We recommend initiating early mobilization using a standardized protocol for patients requiring mechanical ventilation for more than 24 hours to improve outcomes and to reduce ICU costs. However, these protocols may involve additional efforts in an already busy ICU staff and may limit other important care priorities provided by nurses. It may not be feasible to initiate these protocols early in a high percentage of
ICU patients due to the severity of their underlying illnesses. Additionally, it is unclear how much early mobilization would affect unplanned extubation rates. More trials are needed to determine program details and which patients are more likely to benefit.

5. Should patients requiring mechanical ventilation for more than 24 hours be managed with a ventilator liberation protocol?

This panel concluded that patients should be managed with a ventilator liberation protocol. This conclusion was based on randomized control trials which demonstrated a decrease in the number of hours of mechanical ventilation (25 hours; $95 \% \mathrm{Cl}$ : 47 to 12 hours) and a decrease in the length of ICU stay (1 day; $95 \% \mathrm{Cl}: 1.7$ days to 0.2 days). The information on the duration of mechanical ventilation was based on 14 randomized controlled trials with 2205 patients. Eight of these trials involved personnel driven spontaneous breathing trials.

We recommend using a standardized protocol to evaluate for patients' preparedness for liberation from mechanical ventilation. This protocol includes daily evaluation and participation by physicians, nurses, and respiratory therapists. The potential benefits of reduced ICU length of stay and reduced number of hours of mechanical ventilation likely reflect better ICU organization.

6. Should a cuff leak test be performed before extubation of mechanically ventilated adults? Should systemic steroids be administered to patients who fail a cuff leak test before extubation?

This panel suggested that clinicians should perform a cuff leak test on mechanically ventilated adults who meet extubation criteria but who are considered at high risk for post- extubation stridor and/or reintubation. Risk factors for post-extubation stridor include traumatic intubation, prolonged intubation for more than 6 days, large endotracheal tubes, female gender, and reintubation after self extubation. This recommendation was based on a simulation using results from 17 observational studies. This analysis suggested that cuff leak test guided management decreased the rate of reintubation from $4.2 \%$ to $2.4 \%$. The panel does not make suggestions regarding the performance of the cuff leak test or the definition of a positive cuff leak 
test. The panel also suggested that patients who fail a cuff leak test should receive systemic corticosteroids for at least 4 hours prior to extubation. This approach reduces the frequency of post-extubation stridor and reintubation. Since corticosteroids are usually administered for a limited time period, the risks associated with these drugs are modest.

We recommend performing a cuff leak test prior to extubation only in patients at high risk of post-extubation stridor. Patients without a cuff leak should receive IV methylprednisolone at least $4 \mathrm{hrs}$ prior to planned extubation. Cuff leak tests should not be used in all patients or postpone extubation, since extubation can still be successful in many patients without a leak. This often occurs in two groups of patients: women and patients with large endotracheal tubes relative to laryngeal dimensions.

In summary, management of patients requiring mechanical ventilation who are near extubation requires a systematic plan with continuous evaluation of the patient's clinical and respiratory status, judicious use of sedation and analgesia, daily spontaneous breathing trials, early attention to the physical and mental status with rehabilitation, and the use of cuff leak tests.

Keywords: mechanical ventilation, extubation, sedation, cuff leak test
Submitted: 4/11/2017

Author affiliations: Department of Internal Medicine at Texas Tech University Health Sciences Center in Lubbock, TX.

Conflicts of interest: none

\section{REFERENCES}

1. Schmidt GA, Girard TD, Kress JP, et al. Official Executive Summary of an American Thoracic Society/American College of Chest Physicians Clinical Practice Guideline: Liberation from Mechanical Ventilation in Critically Ill Adults. Am J Respir Crit Care Med 2017;195:115-9.

2. Ouellette DR, Patel S, Girard TD, et al. Liberation From Mechanical Ventilation in Critically Ill Adults: An Official American College of Chest Physicians/American Thoracic Society Clinical Practice Guideline: Inspiratory Pressure Augmentation During Spontaneous Breathing Trials, Protocols Minimizing Sedation, and Noninvasive Ventilation Immediately After Extubation. Chest 2017;151:166-80.

3. Girard TD, Alhazzani W, Kress JP, et al. An Official American Thoracic Society/American College of Chest Physicians Clinical Practice Guideline: Liberation from Mechanical Ventilation in Critically Ill Adults. Rehabilitation Protocols, Ventilator Liberation Protocols, and Cuff Leak Tests. Am J Respir Crit Care Med 2017;195:120-33. 